Published in final edited form as:

Int J Psychophysiol. 2011 December ; 82(3): 225-232. doi:10.1016/j.ijpsycho.2011.09.002.

\title{
The interactive effect of change in perceived stress and trait anxiety on vagal recovery from cognitive challenge
}

\author{
Olga V. Crowley, Ph.D., \\ Columbia University Medical Center, Department of Psychiatry, Division of Behavioral Medicine. \\ 1150 St. Nicholas Ave Suite 121 New York, NY 10032 UNITED STATES os2184@columbia.edu
}

Paula S. McKinley, Ph.D.,

Columbia University Medical Center, Department of Psychiatry, Division of Behavioral Medicine. 1150 St. Nicholas Ave Suite 121 New York, NY 10032 UNITED STATES pm491@columbia.edu

Matthew M. Burg, Ph.D.,

Yale University School of Medicine, New Haven, CT; Columbia University School of Medicine, Center for Behavioral and Cardiovascular Health. 630 West 168th Street New York, NY 10032 UNITED STATES mb2358@columbia.edu

Joseph E. Schwartz, Ph.D.,

State University of New York Stony Brook, Department of Psychiatry 101 Nicolls Road 127

Putnam Hall Stony Brook, NY 11794-8790 UNITED STATES Joseph.Schwartz@stonybrook.edu

Carol D. Ryff, Ph.D.,

Department of Psychology, University of Wisconsin, Madison; Institute on Aging and Adult Life, University of Wisconsin, Madison. 1202 West Johnson St. Madison, WI 53706-1969 UNITED

STATES cryff@wisc.edu

Maxine Weinstein, Ph.D.

Center for Population and Health, Georgetown University. 4000 Reservoir Road NW Suite 120 Washington, DC 20007 UNITED STATES weinstma@georgetown.edu

Teresa E. Seeman, Ph.D., and

Division of Geriatrics, UCLA David Geffen School of Medicine, Los Angeles, CA. David Geffen

School of Medicine at UCLA 10945 Le Conte Avenue, Suite 2339 Los Angeles, CA 90095

UNITED STATES tseeman@mednet.ucla.edu

Richard P. Sloan, Ph.D.

Columbia University Medical Center, Department of Psychiatry, Division of Behavioral Medicine, New York, NY. 1150 St. Nicholas Ave Suite 121 New York, NY 10032 UNITED STATES

rps7@columbia.edu

\section{Abstract}

(C) 2011 Elsevier B.V. All rights reserved.

The correspondence should be addressed to: Olga V. Crowley, Ph.D. Columbia University Medical Center, Department of Psychiatry, Division of Behavioral Medicine. 1150 St. Nicholas Ave Suite 121 New York, NY 10032 UNITED STATES os2184@columbia.edu Phone: 212-851-5573 Fax: 212-851-5580.

Publisher's Disclaimer: This is a PDF file of an unedited manuscript that has been accepted for publication. As a service to our customers we are providing this early version of the manuscript. The manuscript will undergo copyediting, typesetting, and review of the resulting proof before it is published in its final citable form. Please note that during the production process errors may be discovered which could affect the content, and all legal disclaimers that apply to the journal pertain. 
The present study tested the hypothesis that the change in state negative affect (measured as perceived stress) after cognitive challenge moderates the relationship of trait anxiety and anger to vagal recovery from that challenge.

Cardiac vagal control (assessed using heart rate variability) and respiratory rate were measured in a sample of 905 participants from the Midlife in the United States Study. Cognitive challenges consisted of computerized mental arithmetic and Stroop color-word matching tasks. Multiple regression analyses controlling for the effects of the demographic, lifestyle, and medical factors influencing cardiac vagal control showed a significant moderating effect of change in perceived stress on the relationship of trait anxiety to vagal recovery from cognitive challenges (Beta $=.253$, $\mathrm{p}=.013$ ). After adjustment for respiratory rate, this effect became marginally significant (Beta $=$. $177, \mathrm{p}=.037)$. In contrast, for the relationship of trait anger to vagal recovery, this effect was not significant either before $($ Beta $=.141, \mathrm{p}=.257)$ or after $(\mathrm{Beta}=.186, \mathrm{p}=.072)$ adjusting for respiratory rate. Secondary analyses revealed that among the individuals with higher levels of trait anxiety, greater reductions in perceived stress were associated with greater increases in cardiac vagal control after the challenge. In contrast, among the individuals with lower levels of trait anxiety, changes in perceived stress had no impact on vagal recovery. Therefore, change in perceived stress moderates the relationship of trait anxiety, but not trait anger, to vagal recovery from cognitive challenge.

\section{Keywords}

state negative affect; perceived stress; trait anxiety; trait anger; heart rate variability recovery; respiratory rate

\section{Introduction}

Trait anxiety and trait anger are established risk factors for incident hypertension and coronary heart disease, and for all-cause and cardiovascular mortality (Chida \& Steptoe, 2009; Denollet \& Pedersen, 2009; Kubzansky, Cole, Kawachi, Vokonas, \& Sparrow, 2006; Rutledge \& Hogan, 2002). One pathway linking these factors to cardiovascular health outcomes may involve the cardiovascular response to psychological stress. Specifically, both exaggerated (Krantz \& Manuck, 1984; Matthews et al., 2004; Treiber et al., 2003) and blunted (Carroll \& Phillips, 2010; Phillips, Der, \& Carroll, 2009) cardiovascular reactivity to psychological stress, and delayed cardiovascular recovery from this stress (Heponiemi et al., 2007; Steptoe \& Marmot, 2006; Stewart, Janicki, \& Kamarck, 2006) predict adverse health outcomes. Evidence suggests that the predictive capacity of cardiovascular recovery from psychological stress may be stronger than that of cardiovascular reactivity (Gerin \& Pickering, 1995; Stewart, et al., 2006). HR recovery from psychological stress is vagally mediated (Mezzacappa, Kelsey, Katkin, \& Sloan, 2001), and cardiac vagal control is an established predictor of cardiovascular morbidity and mortality (Airaksinen, 1999; Kleiger, Miller, \& Bigger Jr, 1987; La Rovere, Bigger Jr, Marcus, Mortara, \& Schwartz, 1998; Tsuji et al., 1996). Thus, vagal recovery from psychological stress has important prognostic implications.

Previous studies investigating the association between trait anxiety, trait anger, and cardiovascular response (e.g., reactivity and recovery) to psychological stress have produced inconsistent results. While some investigators have reported that individuals with higher levels of trait anxiety have blunted cardiovascular (e.g., HR, systolic/diastolic blood pressure [SBP/DBP]) reactivity and delayed recovery (de Rooij, Schene, Phillips, \& Roseboom, 2010; Girdler, Jamner, \& Shapiro, 1997; Gonzalez-Bono et al., 2002; Gramer \& Sprintschnik, 2008; Vitaliano, Russo, Paulsen, \& Bailey, 1995), others have reported no 
association between trait anxiety and cardiovascular response to psychological stress (Jorgensen \& Zachariae, 2006; Knepp \& Friedman, 2008; Ottaviani, Shapiro, Davydov, Goldstein, \& Mills, 2009; Schwerdtfeger, 2004). Similarly, some studies have linked higher levels of trait anger to exaggerated HR, BP (Burns, Bruehl, \& Caceres, 2004; Ratnasingam $\&$ Bishop, 2007), and vagal (Ottaviani, et al., 2009) reactivity, and delayed DBP recovery (Vitaliano, et al., 1995), while others have linked higher levels of trait anger to blunted SBP reactivity (Laude, Girard, Consoli, Mounier-Vehier, \& Elghozi, 1997) and found no association between trait anger and overall cardiovascular recovery (Lache, Meyer, \& Herrmann-Lingen, 2007). One possible reason for the inconsistency in these previously reported findings may be heterogeneity across studies. Specifically, previous studies differ in several critical dimensions, including the measures used to assess the trait characteristic, the samples studied, and the types of laboratory stressors utilized (de Rooij, et al., 2010; Girdler, et al., 1997; Gonzalez-Bono, et al., 2002; Gramer \& Sprintschnik, 2008; Jorgensen \& Zachariae, 2006; Knepp \& Friedman, 2008; Lache, et al., 2007; Laude, et al., 1997; Ottaviani, et al., 2009; Ratnasingam \& Bishop, 2007; Schwerdtfeger, 2004; Vitaliano, et al., 1995). The differences in the study samples represent a particularly important issue as some studies used small samples (Girdler, et al., 1997; Gonzalez-Bono, et al., 2002; Gramer \& Sprintschnik, 2008; Jorgensen \& Zachariae, 2006; Laude, et al., 1997; Schwerdtfeger, 2004) that were limited to either male (Girdler, et al., 1997) or female (Gonzalez-Bono, et al., 2002; Gramer \& Sprintschnik, 2008; Ratnasingam \& Bishop, 2007) participants, while reports based on large samples tended to have limited age range (de Rooij, et al., 2010; Ratnasingam \& Bishop, 2007; Vitaliano, et al., 1995).

Alternatively, the inconsistency may be explained by the inherent lack of evidence regarding whether trait anxiety or trait anger alone are sufficient to generate a physiological response in the laboratory. It is possible that the stressfulness of the task - e.g., the degree to which the stressor elicits an increase in state negative affect, and the speed with which this state resolves after the stressor has ended, also may be important. For example, studies have demonstrated that state negative affect induced by laboratory stress is a strong predictor of the consequent cardiovascular reactivity and recovery (Demaree, Schmeichel, Robinson, \& Everhart, 2004; Feldman, Cohen, Hamrick, \& Lepore, 2004; Gramer \& Sprintschnik, 2008; McClelland, Jones, \& Douglas Gregg, 2009). Here too however, the findings are not fully consistent as some studies have found no association between state negative affect, and either cardiovascular reactivity or recovery (Gramer \& Saria, 2007; Papousek et al., 2010; Schwerdtfeger, 2004).

In summary, previous studies investigating the association between trait anxiety and anger and cardiovascular reactivity to and recovery from psychological stress produced inconsistent results with some studies reporting significant associations (Burns, et al., 2004; de Rooij, et al., 2010; Girdler, et al., 1997; Gonzalez-Bono, et al., 2002; Gramer \& Sprintschnik, 2008; Laude, et al., 1997; Ottaviani, et al., 2009; Ratnasingam \& Bishop, 2007; Vitaliano, et al., 1995) and some studies reporting none (Jorgensen \& Zachariae, 2006; Knepp \& Friedman, 2008; Lache, et al., 2007; Ottaviani, et al., 2009; Schwerdtfeger, 2004). Similarly, the literature that evaluated the links between state negative affect and cardiovascular reactivity and recovery is contradictory (Demaree, et al., 2004; Feldman, et al., 2004; Gramer \& Saria, 2007; Gramer \& Sprintschnik, 2008; McClelland, et al., 2009; Papousek, et al., 2010; Schwerdtfeger, 2004). Methodological heterogeneity among the studies, especially reliance on samples that were limited in their size or demographic representativeness may explain these inconsistencies. Moreover, it may be possible that state negative affect may impact cardiovascular response to stress only against the background of high state negative affect. In other words, state negative affect may moderate (Kraemer, Kiernan, Essex, \& Kupfer, 2008) the association between trait negative affect and the cardiovascular stress response. 
The goal of the present study was therefore to test the hypothesis that state negative affect moderates the relationship of trait negative affect (trait anxiety and anger) to vagal recovery from challenge. We also investigated whether the hypothesized moderating effect is specific to either trait anxiety or anger, or whether this effect is non-specific and may be generalized to the both types of trait negative affect.

\section{Method and materials}

\subsection{Participants}

The data for the current study are from MIDUS II, a 9-year follow-up of the MIDUS I cohort. MIDUS is a national study of midlife development in the United States. MIDUS II included four new studies, one of which, the Biomarker Project conducted from December 2004 to March 2009, included a laboratory-based psychophysiology protocol, from which the current data were drawn. The detailed description of MIDUS study is available elsewhere (Love, Seeman, Weinstein, \& Ryff, 2010; Radler \& Ryff, 2010).

\subsection{Procedures}

Participants traveled to one of three regional sites (Georgetown University, UCLA, or University of Wisconsin, Madison) for an overnight stay in a General Clinical Research Center. The measures of trait anxiety and anger (described below) were completed by the participant in the evening of their arrival. The following morning after a light breakfast with no caffeinated beverages, the psychophysiology protocol was administered. The patient reported to the study room. ECG electrodes were placed on the left and right shoulders, and in the left lower quadrant. The participant was seated, and a keypad for responding to the stress tasks was secured in a comfortable position relative to the dominant hand. Respiration was monitored by inductive plethysmography using the Inductotrace Respiration Monitor (Ambulatory Monitoring, Inc., Ardsley, NY). To measure respiration, stretch bands were placed around the participant's chest and abdomen. Analog signals from chest and abdomen bands were digitized at $20 \mathrm{~Hz}$.

The protocol order (see Fig. 1) was: seated baseline (11 minutes); cognitive challenge 1 (mental arithmetic or Stroop task - 6 minutes); recovery 1 (6 minutes); cognitive challenge 2 (mental arithmetic or Stroop task - 6 minutes); recovery 2 (6 minutes). Task order was counterbalanced. Participants were instructed to remain silent throughout the procedures.

\section{Cognitive stressors}

Mental arithmetic task: A computer-administered mental arithmetic task (Turner et al., 1986) was utilized. The participant was presented with addition or subtraction problems on the computer monitor. After the problem appeared, the participants saw the word "equals" followed by an answer to that problem. The participants' task was to determine if the answer was correct or incorrect by pressing "Yes" or "No" on the keypad within 1 second. The level of difficulty was adjusted based on their performance. The participants were told that their performance was being evaluated in terms of both speed and accuracy.

Stroop color-word matching task: In this computer-administered version of the Stroop task, a color name (blue, green, yellow, or red) was presented on the computer monitor in a color that was either congruent or incongruent with the name. During the task, the keyboard map of the colors appeared at the bottom of each screen. The participants' task was to press a key on the keypad corresponding to the color in which the word was presented rather than the color name. To standardize the level of engagement, the rate of presentation of the stimuli increased as participants performed better and decreased with poorer performance. 
The participants were told that their performance was evaluated in terms of both speed and accuracy.

Evaluation of trait and state negative affect-Trait anxiety was measured using the Spielberger Trait Anxiety Inventory (C. D. Spielberger, 1983). This scale consists of 20 items assessed on a 4-point Likert scale. Higher scores indicate greater trait anxiety. The scale had excellent reliability (Cronbach's [alpha] $=0.904)$.

Trait anger was measured using the Spielberger Trait Anger Inventory (C. D. Spielberger, 1996). This scale consists of 15 items assessed on a 4-point Likert scale. Scale scores were computed by summing across all items. Higher scores indicate greater trait anger. The scale had excellent reliability (Cronbach's [alpha] $=0.810$ ).

State negative affect was measured using participants' self-reported perceived stress ratings. Prior to the beginning of the psychophysiology protocol, the participants were instructed that they would need to rate their stress levels. Specifically, the experimenter said "periodically, during the session I will ask you for a stress rating, which will be on the scale of 1-10 (1 being not stressed at all and 10 being extremely stressed). I will ask: 'may I have a stress rating please.' Then you will give me a number from 1-10 indicating your stress level at that given moment. Just give me the number. Don't elaborate." The change in perceived stress from challenge to recovery was computed by subtracting the averaged stress ratings for the two recovery periods from the averaged ratings for the two challenge periods. Thus, a greater score indicates a greater reduction in perceived stress. The means and the measures of variability for the challenge and recovery perceived stress ratings, and for the change in perceived stress are described in Table 2.

\subsection{Determination of cardiac vagal control}

Following standard procedures we have reported previously (Shcheslavskaya et al., 2010), analog ECG signals were digitized at $500 \mathrm{~Hz}$ by a National Instruments A/D board and passed to a microcomputer for collection. The ECG waveform was submitted to an R-wave detection routine implemented by proprietary event detection software (Graphical Marking [Gmark], author Delano McFarlane, PhD), resulting in an RR interval series. Errors in marking R-waves were corrected interactively (Berntson et al., 1997; Dykes et al., 1986). The RR interval series were then submitted to the software that calculated the standard time and frequency domain indices of HRV (Spectral V2, author Delano McFarlane, PhD). Previous studies have shown that rMSSD of the RR interval time series positively correlates with cardiac vagal control (Berntson, et al., 1997; Kleiger et al., 1991). The rMSSD data were calculated based on 1-minute epochs to allow for the adjustment for respiratory rate. Because rMSSD data was skewed, natural log transformation was performed prior to the analyses.

\subsection{Respiration}

Chest and abdominal respiration signals were submitted to the proprietary software (Spectral V2, author Delano McFarlane, $\mathrm{PhD}$ ) that scored respiration and produced minute-by-minute means of respiratory rate.

\section{5. Assessment of vagal recovery}

To obtain a stable response estimate and to enhance the reliability of our findings, we followed an established procedure recommended in the psychophysiological literature (Kamarck, 1992) and averaged ln rMSSD data for both challenges (mental arithmetic and Stroop tasks), associated recovery periods, and minutes 5 to 10 of the baseline period. 
Vagal recovery was evaluated as a difference between the recovery period and the challenge period. Specifically, a vagal recovery score was computed by subtracting aggregated ln rMSSD during the mental arithmetic and Stroop challenges from the aggregated ln rMSSD during the two associated recovery periods. As cardiac vagal control decreases in response to stress and increases during recovery (Mezzacappa et al., 2001), a greater vagal recovery score represents larger post-stress increases in ln rMSSD.

\subsection{Statistical analyses}

The data were analyzed using SPSS PASW (Predictive Analytics Software, version 18) and SAS (Statistical Analysis Software, version 9.2). All analyses were conducted separately for trait anxiety and trait anger. As there were two types of trait negative affect, Bonferroni corrections were used to control for Type I error with the alpha level of .05/2 $=\mathbf{. 0 2 5}$.

Using multiple linear regression, we tested a model that used main effects of trait negative affect, change in perceived stress, and the interaction of these variables as predictors of vagal recovery. In this model, trait negative affect $x$ change in perceived stress interaction evaluated the moderating effect of change in perceived stress on the relationship of trait affect to vagal recovery. The model that used vagal recovery score as a measure of recovery also controlled for the effect of vagal reactivity (assessed as a delta score computed by subtracting averaged $\ln$ rMSSD during the challenges from the baseline ln rMSSD).

All analyses controlled for perceived stress ratings before and during the challenges, along with demographic, life-style, and medical factors that may influence cardiovascular functioning (described in Table 1). Three dummy variables classifying participants' smoking status were created; two of them (current smoker and ex-smoker) were entered in the model, while the third (never smoked) was used as a reference category. Menopausal status was classified as pre-, peri- and post-menopausal; pre-menopausal status served as a reference. Three types of exercise/physical activity were evaluated separately in MIDUS II: the participants reported how many hours per week they spent performing vigorous, moderate, and light physical activity or exercise. Therefore, we created three continuous exercise/ physical activity variables for the present analysis. The participants who did not report a given type of physical activity were scored as zero. The diseases and medications that can alter cardiac autonomic control were entered in the analysis as covariates. Finally, we also controlled for each participant's sex and BMI.

As heart rate variability is known to be influenced by respiration (Allen, Chambers, \& Towers, 2007; Grossman, Karemaker, \& Wieling, 1991; Grossman \& Taylor, 2007; Grossman, Wilhelm, \& Spoerle, 2004), we conducted all analyses before and after adjusting for respiratory rate. To estimate the variance in ln rMSSD that cannot be explained by the effect of respiration, we conducted within-subject regression analyses using respiratory rate as a predictor of $\ln$ rMSSD on a minute-by-minute basis (Cyranowski, Hofkens, Swartz, Salomon, \& Gianaros, 2011; Sloan et al., 2001). Specifically, separately for each participant, we regressed respiratory rate for each 1-minute epoch on $\ln$ rMSSD for the same epoch (controlling for the effect of the experimental periods, e.g., baseline, challenge, and recovery). We used the resulting unstandardized residual scores as an estimate of the variance in $\ln$ rMSSD that cannot be explained by the effect of respiratory rate. These residuals were then used to compute vagal recovery scores for the respiration-adjusted analyses. 


\section{Results}

\subsection{Sample and measures}

Among 1255 MIDUS II Biomarker Study participants, a total of 1154 individuals had ln rMSSD data, 26 of whom did not complete the described protocol. In addition to these 26 participants, we excluded the city-specific subsample of 183 respondents from Milwaukee, Wisconsin. Among the remaining 945 participants who had ln rMSSD data during at least one of the experimental periods (baseline, mental arithmetic and Stroop tasks, and the two recovery periods), a total of 905 participants had vagal recovery scores (40 participants had missing data for either aggregated Challenge period or aggregated Recovery period). Table 1 provides description of the demographic, life-style, and medical factors for these 905 participants.

Paired-samples t-tests revealed that aggregated ln rMSSD for the mental arithmetic and Stroop tasks elicited a mean withdrawal of $-.139+/-.289(\mathrm{t}(1,889)=14.336, \mathrm{p}=.000)$, mean increase in respiratory rate of $3.706+/-2.238 \mathrm{cpm}(\mathrm{t}(1,877)=-49.075, \mathrm{p}=.000)$, and mean perceived stress increase of $2.696+/-1.751(\mathrm{t}(1,898)=46.171, \mathrm{p}=.000)$. Table 2 provides further description of the means and measures of variability for perceived stress and trait negative affect. Pearson correlations (Table 3) revealed that neither trait anxiety nor anger significantly correlated with change in perceived stress. Trait anxiety and anger were significantly positively correlated. Trait anxiety was associated with significantly higher perceived stress during baseline, stress, and recovery periods. Trait anger was associated with significantly higher perceived stress during the stressors, but it was unrelated to perceived stress during baseline and recovery (Table 3).

\subsection{The moderating effect of change in perceived stress on the relationship of trait anxiety to vagal recovery}

The model that included trait anxiety, change in perceived stress, and their interaction (controlling for vagal reactivity and the demographic, life-style, and medical factors) explained $50.6 \%$ of the variance in vagal recovery $(\mathrm{F}(17,859)=51.77, \mathrm{p}<.0001$; R-Square $=.506$ ). Table 4.1 shows significance tests and regression coefficients for all predictors included in the model. There was a significant moderating effect of change in perceived stress on the relationship of trait anxiety to vagal recovery as indicated by the significant (Beta $=.253, \mathrm{p}=.013)$ corresponding interaction.

To understand the nature of this moderating effect, we examined the relationship of change in perceived stress to vagal recovery among the individuals with higher and lower levels of trait anxiety (based on the median split with the trait anxiety score of 32; see Table 2). We re-ran the models using trait anxiety as a categorical variable to estimate the slopes and the intercepts for the higher- and lower- trait anxiety groups, with the two intercepts centered on the grand mean. In this model, the interaction of trait anxiety median split $\mathrm{x}$ change in perceived stress marginally significantly predicted vagal recovery $(p=0.045)$. The two slopes resulting from this effort visually portrayed the differential strength with which change in perceived stress moderated vagal recovery across the higher- and lower-trait anxiety groups.

Fig. 2.1 illustrates this moderating effect of change in perceived stress on the relationship between trait anxiety and vagal recovery. The participants with higher trait anxiety and smaller reduction or increase in perceived stress seemed to have the smallest increase in $\ln$ rMSSD from the stressor to the recovery period in the entire sample, while the participants with higher trait anxiety and larger reduction in perceived stress seemed to have In rMSSD increases that were similar or even higher than those of the participants who had lower trait 
anxiety ( $\mathrm{p}=.029)$. In contrast, change in perceived stress appeared to have no impact on vagal recovery among participants with lower trait anxiety $(\mathrm{p}=.512)$.

After adjusting for respiratory rate, the results remained the same, although trait anxiety $x$ change in perceived stress interaction became marginally significant $($ Beta $=.177, \mathrm{p}=.037$; see Table 4.1). Fig. 2.2 illustrates the moderating effect of change in perceived stress on the relationship between trait anxiety and vagal recovery after adjusting for respiratory rate.

Like Fig. 2.1, Fig. 2.2 demonstrates that greater reduction in perceived stress was associated with larger ln rMSSD increases (better recovery) among the individuals with higher trait anxiety, but not among their low-anxious counterparts.

\subsection{The moderating effect of change in perceived stress on the relationship of trait anger to vagal recovery}

The model that included trait anger, change in perceived stress, and their interaction (controlling for vagal reactivity and the demographic, life-style, and medical factors that influence cardiovascular functioning) explained $50.3 \%$ of the variance in vagal recovery score $(\mathrm{F}(17,859)=51.10, \mathrm{p}=.000 ; \mathrm{R}-$ Square $=.503)$. Table 4.2 describes significance tests and regression coefficients for all predictors included in the model. The moderational effect of change in perceived stress on the relationship of trait anger to vagal recovery score was not significant $($ Beta $=.141, \mathrm{p}=.257$ ). After adjusting for respiratory rate, this effect remained insignificant $(\mathrm{Beta}=.186, \mathrm{p}=.072)$. Interestingly, the main effect of trait anger was also not significant before (Beta $=-.053, \mathrm{p}=.261)$ and marginally significant after (Beta $=-$. $086, p=.028$ ) adjusting for the effects of the respiratory rate (See Table 4.2).

\section{Discussion}

Our results demonstrated that change in perceived stress from the challenge to the recovery period moderated the association between trait anxiety and vagal recovery. Among the individuals with higher levels of trait anxiety, a smaller reduction - or an increase - in perceived stress was associated with smaller increases in $\ln$ rMSSD, while a greater reduction in perceived stress was associated with larger increases in In rMSSD after the challenge. In contrast, among the individuals with lower levels of trait anxiety, change in perceived stress had little impact on ln rMSSD increases after the challenge. Although after adjustment for respiratory rate the model became marginally significant, the results still revealed the moderating effect of change in perceived stress on the relationship of trait anxiety to vagal recovery.

This moderating effect of change in perceived stress appears to be specific to trait anxiety as the interactive effect of change in perceived stress and trait anger did not predict vagal recovery. The specificity of this moderating effect may be due to the fact that our measure of perceived stress may be more closely related to state anxiety than to state anger. Indeed, trait anxiety significantly correlated with perceived stress ratings during the baseline, stressor, and recovery periods, while trait anger only related to these ratings during the stressors. Moreover, the psychological stressors used in MIDUS II, computerized mental arithmetic and Stroop tasks, differ from the stressors - such as anger recall task or harassment - that are typically employed in research concerning the impact of trait anger on the cardiovascular stress response (Chida \& Hamer, 2008; Gerin, Davidson, Christenfeld, Goyal, \& Schwartz, 2006; Glynn, Christenfeld, \& Gerin, 2002; Gregg, James, Matyas, \& Thorsteinsson, 1999; Ironson et al., 1992). Consistent with this line of reasoning, trait anger was unrelated to vagal recovery from cognitive challenge, and control for respiration did not substantially alter this finding. 
Our findings suggest that trait anxiety alone may not be sufficient to account for vagal responses in the laboratory. Rather, the preexisting trait vulnerability is consequential for vagal response only in the context of high perceived stress. Thus, trait-like vulnerability (at least for anxiety) must be accompanied by ongoing stress to affect vagal recovery following laboratory challenge. Our finding is consistent with the evidence suggesting that state affect moderates the association between trait negative affect and cardiovascular recovery (Souza et al., 2007). Souza et al reported that positive affective priming (exposure to pleasant images) was associated with faster heart period recovery after a public speaking task among individuals with lower levels of trait negative affect, but not among their counterparts with higher levels of trait negative affect. We found that change in perceived stress was associated with faster vagal recovery among individuals with higher levels of trait anxiety, but not among their low-anxious counterparts. Thus, future studies may investigate whether state positive affect predicts cardiovascular recovery among individuals with low levels of trait negative affect, while state negative affect predicts cardiovascular recovery among individuals with high levels of trait negative affect.

Previous studies also have demonstrated the importance of the interactive effects of state and trait negative affect for predicting cardiovascular functioning. For example, Burg et al (Burg, Lampert, Joska, Batsford, \& Jain, 2004) reported that those patients who had sustained ventricular arrhythmias (treated with implanted cardioverter-defibrillators shock) that were triggered by state anxiety and state anger also had higher levels of trait anxiety and trait anger. In contrast, those patients who had sustained ventricular arrhythmias that were not emotion-triggered did not have high levels of trait anxiety and trait anger. Thus, the combination of high state and trait anxiety and anger has negative implications for cardiovascular health (Burg, et al., 2004). Our results suggest that a combination of a large reduction in state negative affect and high trait anxiety may have cardioprotective implications. Indeed, cardiac vagal control is an established predictor of cardiovascular disease (CVD) risk (Airaksinen, 1999; Kleiger, et al., 1987; La Rovere, et al., 1998; Tsuji, et al., 1996). The fact that greater reduction in state negative affect in individuals with high trait anxiety was associated with the rates of vagal recovery that were similar or even faster compared with their counterparts who had low levels of trait anxiety implies that having high levels of trait anxiety alone may not automatically predispose one to the increased CVD risk (as indexed by vagal recovery). Rather, it is important to consider the interactive effects of state and trait anxiety. Therefore, our findings may generate future research elucidating the implications of the interactive effects of state and trait anxiety for the prediction of cardiovascular health outcomes.

Our findings are limited by the use of perceived stress as our index of state negative affect. This is a relatively crude estimate, which does not describe the nature of the affective state experienced by our participants. Thus, we could not evaluate whether perceived stress reflected states of anxiety, anger, or more general negative affect. The inferences about the nature of the state negative affect measured by our index can only be made on a basis of the correlational analysis that revealed that perceived stress ratings correlated with trait anxiety during the baseline, stressor, and recovery periods; in contrast, perceived stress ratings only correlated with trait anger during the stressor period. This "secondary" evidence, however, is not sufficient. Without the established measures of state anxiety and state anger, such as State-Trait Anxiety Inventory or the State-Trait Anger Expression Inventory, it is not possible to determine the specific type of state negative affect experienced by our participants. Therefore, lack of detailed assessment of state anxiety and state anger constitutes a major methodological limitation of our study.

Our findings contribute to the existing literature in two key ways. First, unlike previously published reports that used small study samples (Girdler, et al., 1997; Gonzalez-Bono, et al., 
2002; Gramer \& Sprintschnik, 2008; Jorgensen \& Zachariae, 2006; Laude, et al., 1997; Schwerdtfeger, 2004), were limited to either male (Girdler, et al., 1997) or female (Gonzalez-Bono, et al., 2002; Gramer \& Sprintschnik, 2008; Ratnasingam \& Bishop, 2007) participants, and represented limited age range (de Rooij, et al., 2010; Ratnasingam \& Bishop, 2007; Vitaliano, et al., 1995), we utilized the large, demographically representative MIDUS II data set that included both male and female participants across a wide age spectrum. Second, our approach to analyzing perceived stress represents another methodological strength as we assessed the dynamics of this variable by evaluating the change from the challenge to the recovery period, mirroring the concurrent change in vagal activation.

In summary, the present study demonstrated that the interaction of change in perceived stress with trait anxiety orchestrated vagal recovery from cognitive challenge. This effect was specific to trait anxiety, but not to trait anger. Methodological concerns, such as greater concordance of our measure of perceived stress with trait anxiety than with trait anger, limit the interpretation of our findings. Our findings may generate future research elucidating the role of state negative affect in the association between other types of trait affect and cardiovascular response to stress, and the implications of the regulation of trait anxiety for the prediction of cardiovascular health outcomes.

\section{Acknowledgments}

This study was supported by a grant from the National Institute on Aging (P01-AG020166) to conduct a longitudinal follow-up of the MIDUS (Midlife in the U.S.) investigation (Dr. Ryff). The original study was supported by a grant from the John D. and Catherine T. MacArthur Foundation Research Network on Successful Midlife Development (Dr. Ryff). We thank the staff of the Clinical Research Centers at the University of Wisconsin-Madison (UW), UCLA, and Georgetown University for their support in conducting this study. The study also received support from M01-RR023942 (Georgetown), M01-RR00865 (UCLA) from the General Clinical Research Centers Program and 1UL1RR025011 (UW) from the Clinical and Translational Science Award (CTSA) program of the National Center for Research Resources, National Institutes of Health and from the Nathaniel Wharton Fund. We thank the staff of the Clinical Research Centers at the University of Wisconsin-Madison (UW), UCLA, and Georgetown University for their support in conducting this study.

\section{Glossary}

BP

blood pressure

SBP systolic blood pressure

DBP diastolic blood pressure

HR heart rate

HRV heart rate variability

rMSSD square root of the mean squared differences of successive RR intervals

CVD cardiovascular disease

CHD coronary heart disease

\section{REFERENCES}

Airaksinen KEJ. Autonomic mechanisms and sudden death after abrupt coronary occlusion. Annals of Medicine. 1999; 31(4):240-245. [PubMed: 10480754]

Allen JJB, Chambers AS, Towers DN. The many metrics of cardiac chronotropy: A pragmatic primer and a brief comparison of metrics. Biological Psychology. 2007; 74(2):243-262. [PubMed: 17070982] 
Berntson GG, Bigger J. Thomas Jr, Eckberg DL, Grossman P, Kaufmann PG, Malik M, et al. Heart rate variability: Origins methods, and interpretive caveats. Psychophysiology. 1997; 34(6):623-648. [PubMed: 9401419]

Burg MM, Lampert R, Joska T, Batsford W, Jain D. Psychological traits and emotion-triggering of ICD shock-terminated arrhythmias. Psychosomatic Medicine. 2004; 66(6):898-902. [PubMed: 15564355]

Burns JW, Bruehl S, Caceres C. Anger management style, blood pressure reactivity, and acute pain sensitivity: Evidence for "trait x situation" models. Annals of Behavioral Medicine. 2004; 27(3): 195-204. [PubMed: 15184095]

Carroll, D.; Phillips, A. The health and behavioral corollaries of blunted physiological reactions to acute psychological stress: Revising the reactivity hypothesis. Paper presented at the Amrican Psychosomatic Society 68th Annual Scientific Meeting; 2010.

Chida Y, Hamer M. Chronic Psychosocial Factors and Acute Physiological Responses to LaboratoryInduced Stress in Healthy Populations: A Quantitative Review of 30 Years of Investigations. Psychological Bulletin. 2008; 134(6):829-885. [PubMed: 18954159]

Chida Y, Steptoe A. The Association of Anger and Hostility With Future Coronary Heart Disease. A Meta-Analytic Review of Prospective Evidence. Journal of the American College of Cardiology. 2009; 53(11):936-946. [PubMed: 19281923]

Christenfeld N, Glynn LM, Gerin W. On the reliable assessment of cardiovascular recovery: An application of curve-fitting techniques. Psychophysiology. 2000; 37(4):543-550. [PubMed: 10934913]

Cyranowski JM, Hofkens TL, Swartz HA, Salomon K, Gianaros PJ. Cardiac vagal control in nonmedicated depressed women and nondepressed controls: Impact of depression status, lifetime trauma history, and respiratory factors. Psychosomatic Medicine. 2011; 73(4):336-343. [PubMed: 21364194]

de Rooij SR, Schene AH, Phillips DI, Roseboom TJ. Depression and anxiety: Associations with biological and perceived stress reactivity to a psychological stress protocol in a middle-aged population. Psychoneuroendocrinology. 2010; 35(6):866-877. [PubMed: 20031333]

Demaree HA, Schmeichel BJ, Robinson JL, Everhart DE. Behavioural, affective, and physiological effects of negative and positive emotional exaggeration. Cognition and Emotion. 2004; 18(8): 1079-1097.

Denollet J, Pedersen SS. Anger, Depression, and Anxiety in Cardiac Patients. The Complexity of Individual Differences in Psychological Risk. Journal of the American College of Cardiology. 2009; 53(11):947-949. [PubMed: 19281924]

Dykes FD, Ahmann PA, Baldzer K, Carrigan TA, Kitney RI, Giddens DP. Breath amplitude modulation of heart rate variability in normal full term neonates. Pediatric Research. 1986; 20:301-308. [PubMed: 3703619]

Feldman PJ, Cohen S, Hamrick N, Lepore SJ. Psychological stress, appraisal, emotion and cardiovascular response in a public speaking task. Psychology and Health. 2004; 19(3):353-368.

Gerin W, Davidson KW, Christenfeld NJS, Goyal T, Schwartz JE. The role of angry rumination and distraction in blood pressure recovery from emotional arousal. Psychosomatic Medicine. 2006; 68(1):64-72. [PubMed: 16449413]

Gerin W, Pickering TG. Association between delayed recovery of blood pressure after acute mental stress and parental history of hypertension. Journal of Hypertension. 1995; 13(6):603-610. [PubMed: 7594416]

Girdler SS, Jamner LD, Shapiro D. Hostility, testosterone, and vascular reactivity to stress: Effects of sex. International Journal of Behavioral Medicine. 1997; 4(3):242-263. [PubMed: 16250731]

Glynn LM, Christenfeld N, Gerin W. The role of rumination in recovery from reactivity: Cardiovascular consequences of emotional states. Psychosomatic Medicine. 2002; 64(5):714-726. [PubMed: 12271102]

Gonzalez-Bono E, Moya-Albiol L, Salvador A, Carrillo E, Ricarte J, Gomez-Amor J. Anticipatory autonomic response to a public speaking task in women: The role of trait anxiety. Biological Psychology. 2002; 60(1):37-49. [PubMed: 12100844] 
Gramer M, Saria K. Effects of social anxiety and evaluative threat on cardiovascular responses to active performance situations. Biological Psychology. 2007; 74(1):67-74. [PubMed: 16950557]

Gramer M, Sprintschnik E. Social anxiety and cardiovascular responses to an evaluative speaking task: The role of stressor anticipation. Personality and Individual Differences. 2008; 44(2):371-381.

Gregg ME, James JE, Matyas TA, Thorsteinsson EB. Hemodynamic profile of stress-induced anticipation and recovery. International Journal of Psychophysiology. 1999; 34(2):147-162. [PubMed: 10576399]

Grossman P, Karemaker J, Wieling W. Prediction of tonic parasympathetic cardiac control using respiratory sinus arrhythmia: The need for respiratory control. Psychophysiology. 1991; 28(2): 201-216. [PubMed: 1946886]

Grossman P, Taylor EW. Toward understanding respiratory sinus arrhythmia: Relations to cardiac vagal tone, evolution and biobehavioral functions. Biological Psychology. 2007; 74(2):263-285. [PubMed: 17081672]

Grossman P, Wilhelm FH, Spoerle M. Respiratory sinus arrhythmia, cardiac vagal control, and daily activity. American Journal of Physiology - Heart and Circulatory Physiology. 2004; 287(2 56-2):H728-H734. [PubMed: 14751862]

Heponiemi T, Elovainio M, Pulkki L, Puttonen S, Raitakari O, Keltikangas-Jarvinen L. Cardiac autonomic reactivity and recovery in predicting carotid atherosclerosis: The cardiovascular risk in young Finns study. [Article]. Health Psychology. 2007; 26(1):13-21. [PubMed: 17209693]

Ironson G, Taylor CB, Boltwood M, Bartzokis T, Dennis C, Chesney M, et al. Effects of anger on left ventricular ejection fraction in coronary artery disease. American Journal of Cardiology. 1992; 70(3):281-285. [PubMed: 1632389]

Jorgensen MM, Zachariae R. Repressive coping style and autonomic reactions to two experimental stressors in healthy men and women: Health and Disability. Scandinavian Journal of Psychology. 2006; 47(2):137-148. [PubMed: 16542356]

Kamarck TW. For Distinguished Early Career Contribution to Psychophysiology - Spr Award Address, 1991 - Recent Developments in the Study of Cardiovascular Reactivity - Contributions from Psychometric Theory and Social-Psychology. Psychophysiology. 1992; 29(5):491-503. [PubMed: 1410179]

Kario K, Schwartz JE, Gerin W, Robayo N, Maceo E, Pickering TG. Psychological and physical stress-induced cardiovascular reactivity and diurnal blood pressure variation in women with different work shifts. Hypertension research. 2002; 25(4):543-551. [PubMed: 12358139]

Kleiger RE, Bigger JT, Bosner MS, Chung MK, Cook JR, Rolnitzky LM, et al. Stability over time of variables measuring heart rate variability in normal subjects. American Journal of Cardiology. 1991; 68(6):626-630. [PubMed: 1877480]

Kleiger RE, Miller JP, Bigger JT Jr. Decreased heart rate variability and its association with increased mortality after acute myocardial infarction. American Journal of Cardiology. 1987; 59(4):256262. [PubMed: 3812275]

Knepp MM, Friedman BH. Cardiovascular activity during laboratory tasks in women with high and low worry. Biological Psychology. 2008; 79(3):287-293. [PubMed: 18706472]

Kraemer HC, Kiernan M, Essex M, Kupfer DJ. How and Why Criteria Defining Moderators and Mediators Differ Between the Baron \& Kenny and MacArthur Approaches. Health Psychology. 2008; 27(2 SUPPL. 2):S101-S108. [PubMed: 18377151]

Krantz DS, Manuck SB. Acute psychophysiologic reactivity and risk of cardiovascular disease: A review and methodologic critique. Psychological Bulletin. 1984; 96(3):435-464. [PubMed: 6393178]

Kubzansky LD, Cole SR, Kawachi I, Vokonas P, Sparrow D. Shared and unique contributions of anger, anxiety, and depression to coronary heart disease: A prospective study in the normative aging study. Annals of Behavioral Medicine. 2006; 31(1):21-29. [PubMed: 16472035]

La Rovere MT, Bigger JT Jr, Marcus FI, Mortara A, Schwartz PJ. Baroreflex sensitivity and heart-rate variability in prediction of total cardiac mortality after myocardial infarction. Lancet. 1998; 351(9101):478-484. [PubMed: 9482439] 
Lache B, Meyer T, Herrmann-Lingen C. Social support predicts hemodynamic recovery from mental stress in patients with implanted defibrillators. Journal of Psychosomatic Research. 2007; 63(5): 515-523. [PubMed: 17980225]

Laude D, Girard A, Consoli S, Mounier-Vehier C, Elghozi JL. Anger expression and cardiovascular reactivity to mental stress: A spectral analysis approach. Clinical and Experimental Hypertension. 1997; 19(5-6):901-911. [PubMed: 9247763]

Love GD, Seeman TE, Weinstein M, Ryff CD. Bioindicators in the MIDUS National Study: Protocol, Measures, Sample, and Comparative Context. Journal of Aging and Health. 2010; 22:1059-1080. [PubMed: 20876364]

Matthews KA, Katholi CR, McCreath H, Whooley MA, Williams DR, Zhu S, et al. Blood pressure reactivity to psychological stress predicts hypertension in the CARDIA study. Circulation. 2004; 110(1):74-78. [PubMed: 15210592]

McClelland AB, Jones KV, Gregg M. E. Douglas. Psychological and cumulative cardiovascular effects of repeated angry rumination and visuospatial suppression. International Journal of Psychophysiology. 2009; 74(2):166-173. [PubMed: 19732797]

Mezzacappa ES, Kelsey RM, Katkin ES, Sloan RP. Vagal rebound and recovery from psychological stress. Psychosomatic Medicine. 2001; 63(4):650-657. [PubMed: 11485119]

Ottaviani C, Shapiro D, Davydov DM, Goldstein IB, Mills PJ. The autonomic phenotype of rumination. International Journal of Psychophysiology. 2009; 72(3):267-275. [PubMed: 19272312]

Papousek I, Nauschnegg K, Paechter M, Lackner HK, Goswami N, Schulter G. Trait and state positive affect and cardiovascular recovery from experimental academic stress. Biological Psychology. 2010; 83(2):108-115. [PubMed: 19944130]

Phillips AC, Der G, Carroll D. Self-reported health and cardiovascular reactions to psychological stress in a large community sample: Cross-sectional and prospective associations. Psychophysiology. 2009; 46(5):1020-1027. [PubMed: 19497004]

Radler, BT.; Ryff, CD. Who Participates? Accounting for Longitudinal Retention in the MIDUS National Study of Health and Well-Being. 2010. p. 0898264309358617

Ratnasingam P, Bishop GD. Social support schemas, trait anger, and cardiovascular responses. International Journal of Psychophysiology. 2007; 63(3):308-316. [PubMed: 17275113]

Rutledge T, Hogan BE. A quantitative review of prospective evidence linking psychological factors with hypertension development. Psychosomatic Medicine. 2002; 64(5):758-766. [PubMed: 12271106]

Schwerdtfeger A. Predicting autonomic reactivity to public speaking: Don't get fixed on self-report data! International Journal of Psychophysiology. 2004; 52(3):217-224. [PubMed: 15094245]

Shcheslavskaya OV, Burg MM, McKinley PS, Schwartz JE, Gerin W, Ryff CD, et al. Heart rate recovery after cognitive challenge is preserved with age. Psychosomatic Medicine. 2010; 72(2): 128-133. [PubMed: 20028831]

Sloan RP, Bagiella E, Shapiro PA, Kuhl JP, Chernikhova D, Berg J, et al. Hostility, gender, and cardiac autonomic control. Psychosomatic Medicine. 2001; 63(3):434-440. [PubMed: 11382270]

Souza GGL, Mendonça-De-Souza ACF, Barros EM, Coutinho EFS, Oliveira L, Mendlowicz MV, et al. Resilience and vagal tone predict cardiac recovery from acute social stress. Stress. 2007; 10(4): 368-374. [PubMed: 17853065]

Spielberger, CD. Manual for the state-trait anxiety inventory (Form Y) (“Self-evaluation questionnaire”). Consulting Psychologists Press; Palo Alto, CA: 1983.

Spielberger, CD. State-Trait Anger Expression Inventory: Professional manual. Odessa, FL: 1996.

Steptoe A, Marmot M. Psychosocial, hemostatic, and inflammatory correlates of delayed poststress blood pressure recovery. Psychosomatic Medicine. 2006; 68(4):531-537. [PubMed: 16868261]

Stewart JC, Janicki DL, Kamarck TW. Cardiovascular reactivity to and recovery from psychological challenge as predictors of 3-year change in blood pressure. Health Psychology. 2006; 25(1):111118. [PubMed: 16448304]

Treiber FA, Kamarck T, Schneiderman N, Sheffield D, Kapuku G, Taylor T. Cardiovascular reactivity and development of preclinical and clinical disease states. Psychosomatic Medicine. 2003; 65(1): 46-62. [PubMed: 12554815] 
Tsuji H, Larson MG, Venditti FJ Jr, Manders ES, Evans JC, Feldman CL, et al. Impact of reduced heart rate variability on risk for cardiac events: The Framingham Heart Study. Circulation. 1996; 94(11):2850-2855. [PubMed: 8941112]

Turner JR, Hewitt JK, Morgan RK, Simes J, Carroll D, Kelly KA. Graded mental arithmetic as an active psychological challenge. International Journal of Psychophysiology. 1986; 3:307-309. [PubMed: 3754545]

Vitaliano PP, Russo J, Paulsen VM, Bailey SL. Cardiovascular recovery from laboratory stress: Biopsychosocial concomitants in older adults. Journal of Psychosomatic Research. 1995; 39(3): 361-377. [PubMed: 7636779] 


\section{Highlights}

$>$ We hypothesized that change in perceived stress moderates the effect of trait affect on vagal recovery

> We used MIDUS II data set to test our hypothesis

$>$ We measured trait negative affect in terms of trait anxiety and trait anger

$>$ Change in perceived stress moderated effects of trait anxiety, but not anger, on vagal recovery 


\begin{tabular}{|c|c|c|c|c|}
\hline $\begin{array}{l}\text { Seated } \\
\text { Resting } \\
\text { Baseline }\end{array}$ & $\begin{array}{c}\text { Psychological } \\
\text { Stressor } 1 \\
\text { (Math or Stroop } \\
\text { Task)* }\end{array}$ & Recovery 1 & $\begin{array}{c}\text { Psychological } \\
\text { Stressor } 2 \\
\text { (Math or Stroop } \\
\text { Task) }\end{array}$ & Recovery 2 \\
\hline $11 \mathrm{mins}$ & 6 mins & 6 mins & 6 mins & $6 \mathrm{mins}$ \\
\hline
\end{tabular}

Fig. 1.

Psychophysiology Protocol 


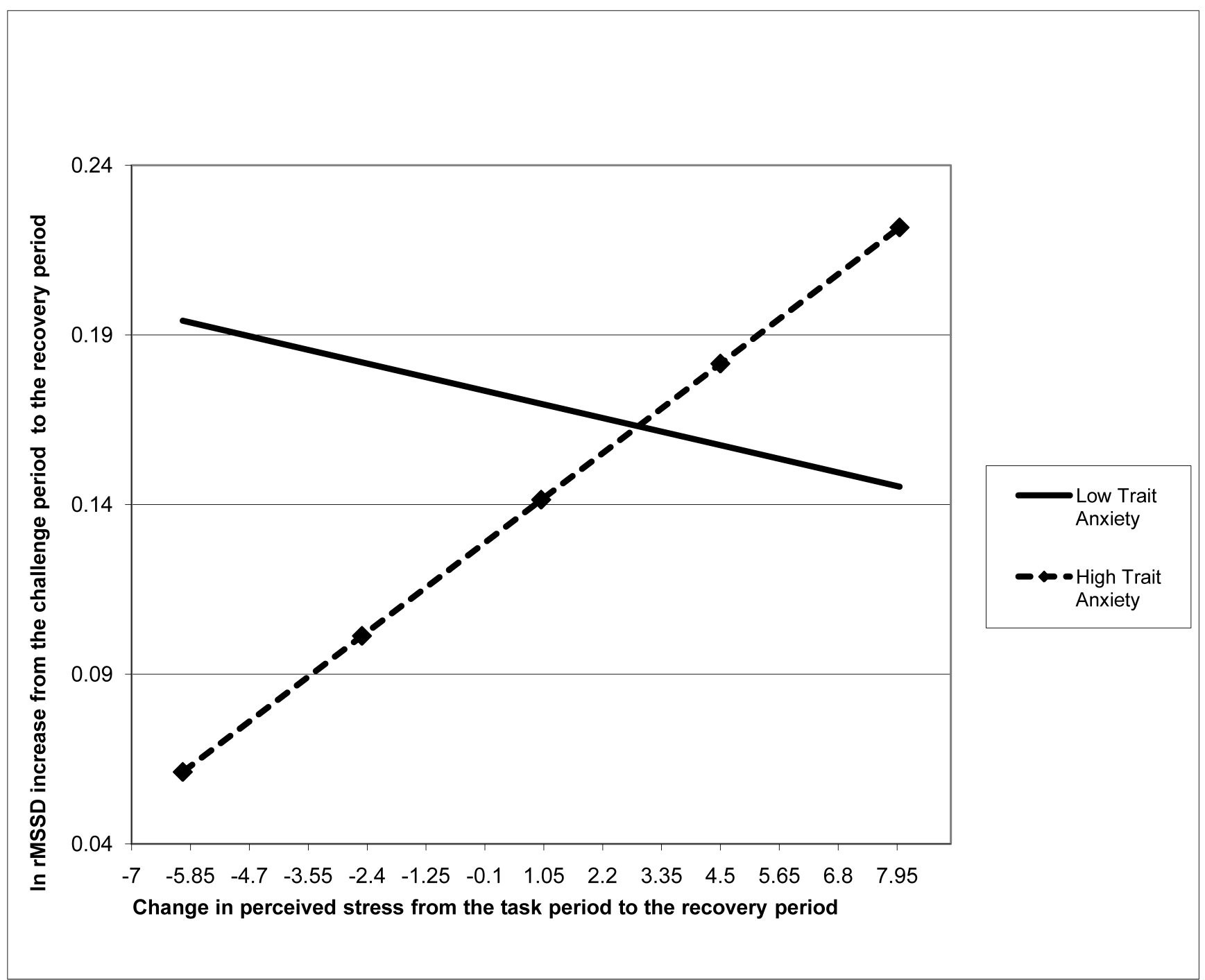

Fig. 2.1.

The moderating effect of change in state negative affect on the relationship of trait anxiety to vagal recovery score: before adjusting for respiratory rate 


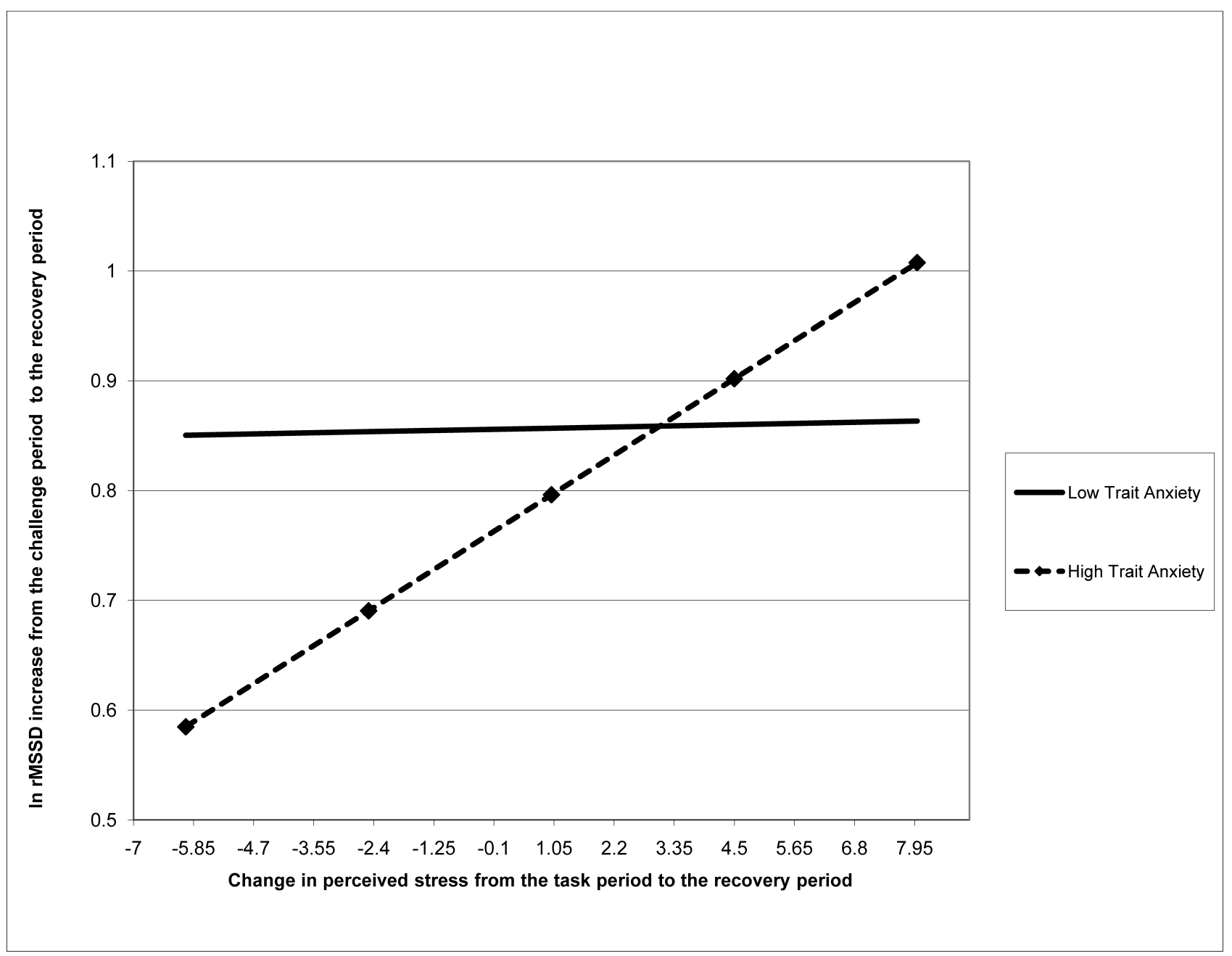

Fig. 2.2.

The moderating effect of change in state negative affect on the relationship of trait anxiety to vagal recovery score: after adjusting for respiratory rate 
Table 1

Sample characteristics

\begin{tabular}{|c|c|c|c|}
\hline Variable & & $\mathbf{N}$ & Mean and standard deviation \\
\hline Age & & 905 & $57.13+/-11.30$ \\
\hline BMI & & 905 & $29.14+/-5.96$ \\
\hline \multirow{10}{*}{$\begin{array}{l}\text { Diseases altering cardiac } \\
\text { autonomic function }\end{array}$} & High Blood pressure & 285 & NA \\
\hline & Heart Disease & 86 & \\
\hline & Circulation problems & 53 & \\
\hline & TIA or stroke & 29 & \\
\hline & Depression & 175 & \\
\hline & Diabetes & 92 & \\
\hline & Cholesterol problems & 379 & \\
\hline & Asthma & 103 & \\
\hline & Emphysema/COPD & 25 & \\
\hline & Thyroid disease & 113 & \\
\hline \multirow{2}{*}{$\begin{array}{l}\text { Medications altering cardiac } \\
\text { autonomic control }\end{array}$} & Yes & 310 & NA \\
\hline & No & 595 & \\
\hline \multirow{2}{*}{$\begin{array}{l}\text { Have any of the diseases/take } \\
\text { any medications listed above }\end{array}$} & Yes & 677 & NA \\
\hline & No & 228 & \\
\hline \multirow[t]{2}{*}{ Sex } & Male & 406 & NA \\
\hline & Female & 499 & \\
\hline \multirow[t]{3}{*}{ Menopausal status } & Pre-menopausal & 145 & NA \\
\hline & Peri-menopausal & 41 & \\
\hline & Post-menopausal & 311 & \\
\hline \multirow[t]{3}{*}{ Smoking } & Never & 506 & NA \\
\hline & Smoker & 104 & \\
\hline & Ex-smoker & 295 & \\
\hline \multirow{3}{*}{$\begin{array}{l}\text { Exercise/physical activity } \\
\text { (hours per week) }\end{array}$} & Vigorous & 905 & $.96+/-2.92$ \\
\hline & Moderate & 905 & $2.83+/-5.61$ \\
\hline & Light & 905 & $1.712+/-4.45$ \\
\hline
\end{tabular}




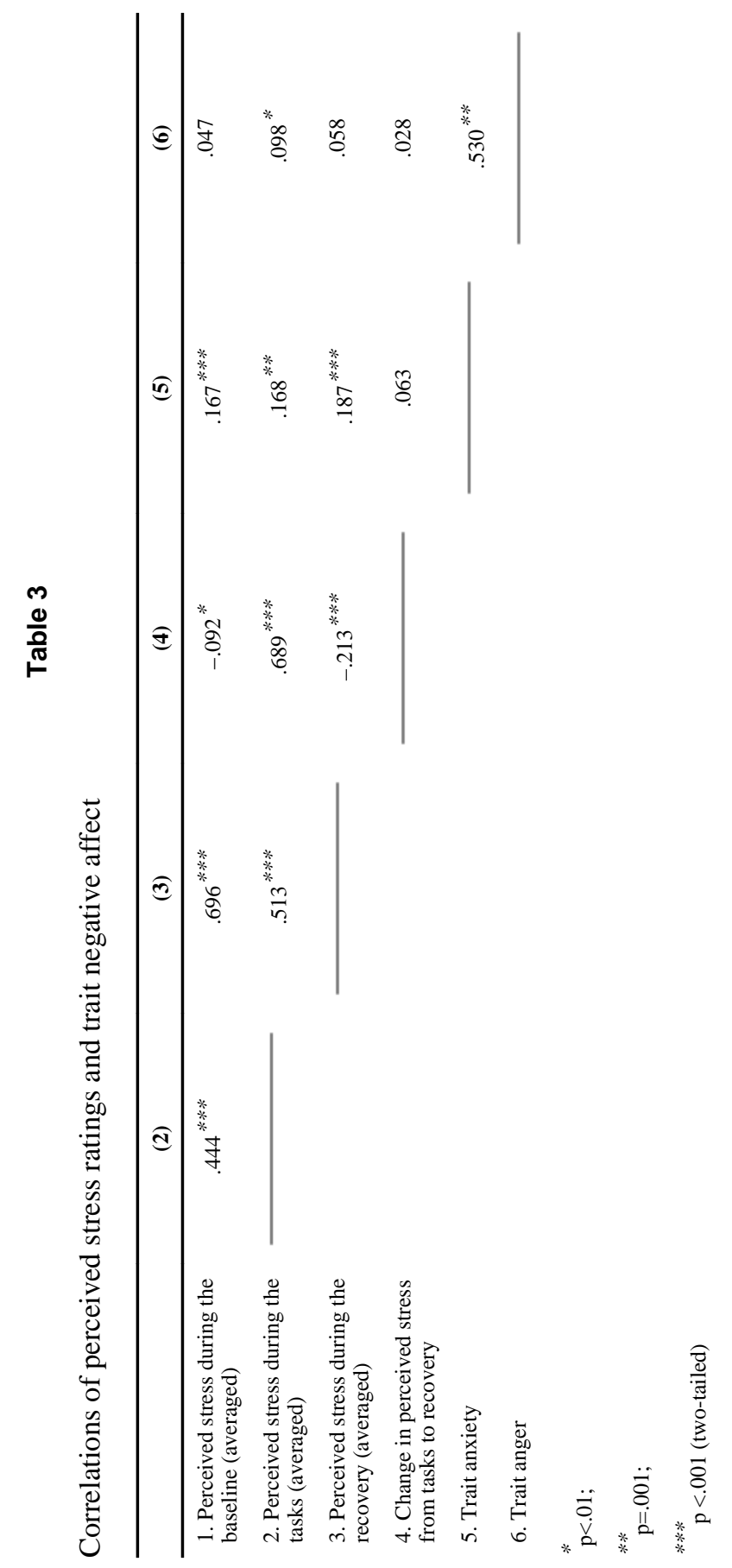

Int J Psychophysiol. Author manuscript; available in PMC 2013 January 30. 


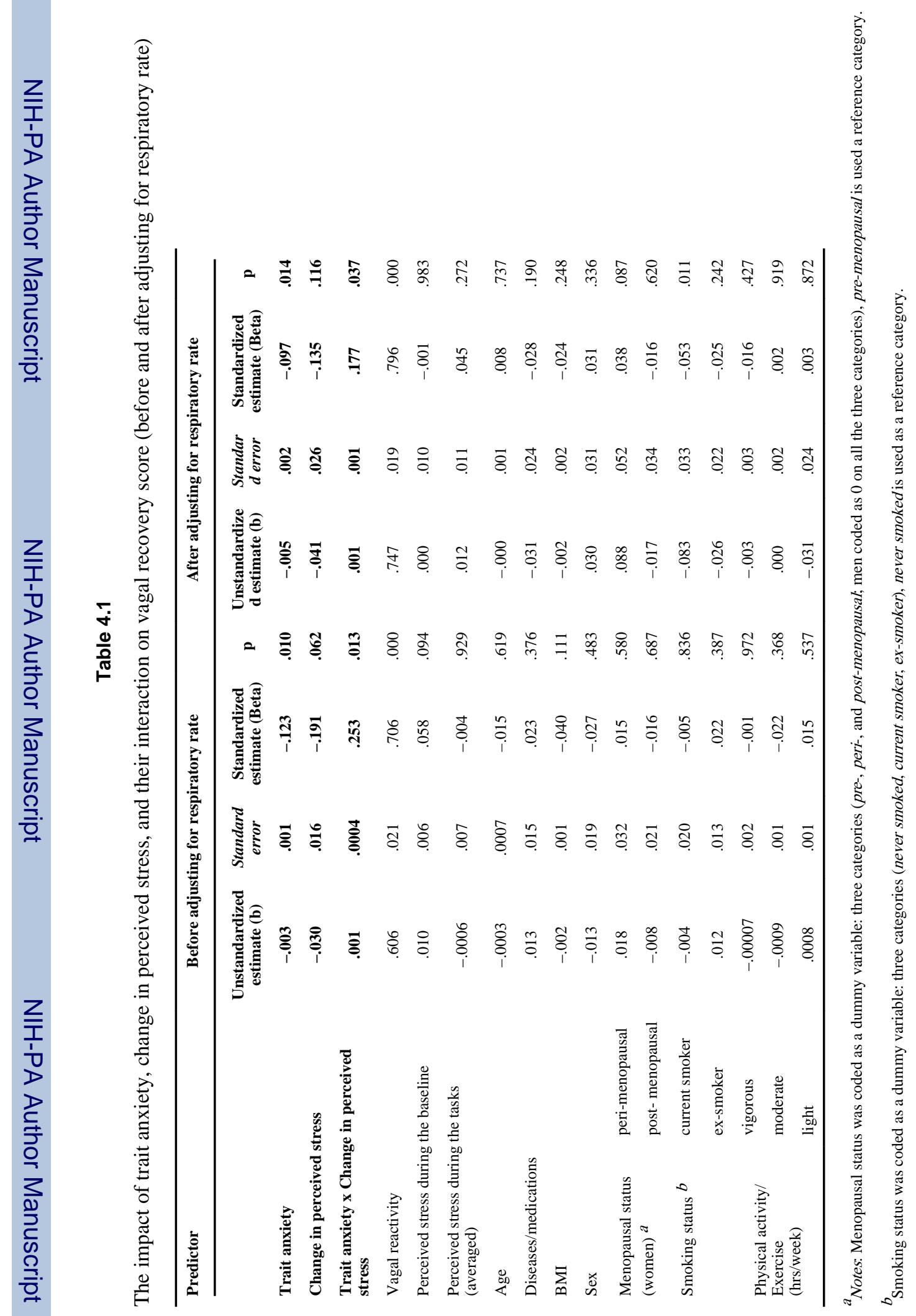

Int J Psychophysiol. Author manuscript; available in PMC 2013 January 30. 


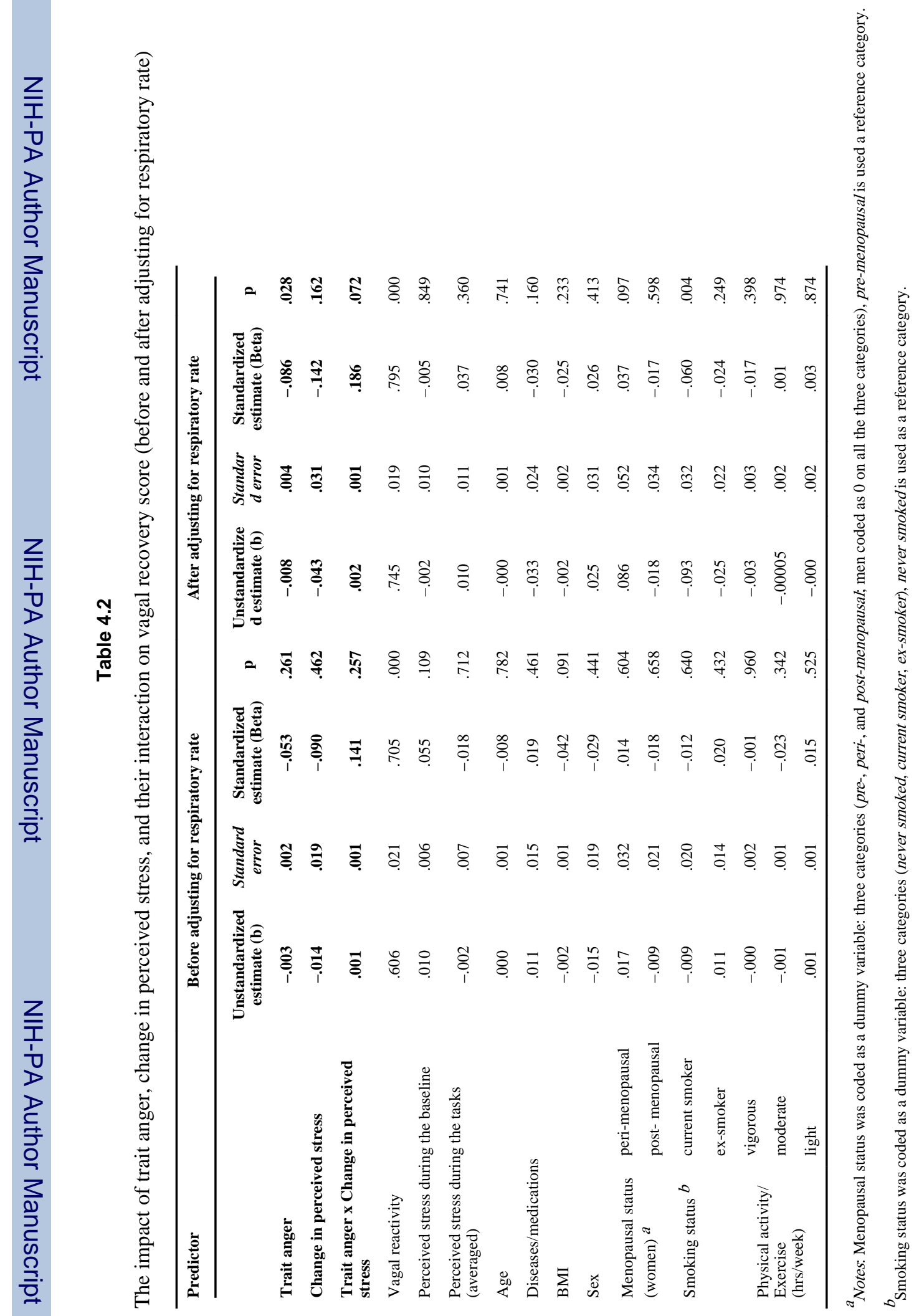

Int J Psychophysiol. Author manuscript; available in PMC 2013 January 30. 